\title{
The role of alternative (advanced) conscious sedation techniques in dentistry for adult patients: a series of cases
}

\author{
N. Robb'
}

\section{VERIFIABLE CPD PAPER}

The basic techniques of conscious sedation have been found to be safe and effective for the management of anxiety in adult dental patients requiring sedation to allow them to undergo dental treatment. There remains great debate within the profession as to the role of the so called advanced sedation techniques. This paper presents a series of nine patients who were managed with advanced sedation techniques where the basic techniques were either inappropriate or had previously failed to provide adequate relief of anxiety. In these cases, had there not been the availability of advanced sedation techniques, the most likely recourse would have been general anaesthesia - a treatment modality that current guidance indicates should not be used where there is an appropriate alternative. The sedation techniques used have provided that appropriate alternative management strategy.

\section{INTRODUCTION}

The use of conscious sedation in dentistry is one of the most widely discussed and regulated areas in dentistry. In the last 20 years there have been multiple publications providing guidance, guidelines and clinical standards for the practice of this area of dentistry. There is the Standing Dental Advisory Committee on Dental Sedation; ${ }^{1}$ Royal College of Anaesthetists; ${ }^{2}$ Academy of Medical Royal Colleges and their Faculties; ${ }^{3}$ Scottish Dental Clinical Effectiveness Programme; ${ }^{4}$ Standing Committee on Sedation for Dentistry; ${ }^{5}$ Dental Sedation Teachers' Group; ${ }^{6}$ and the Independent Expert Group on Training and Standards for Sedation in Dentistry. ${ }^{7-9}$

The terminology used has varied with distinction made between basic or standard and alternative or advanced techniques. The debate is less than helpful as terms tend to be used in a defensive way by proponents of the techniques and in pejorative ways by those who are against the use of the techniques. The basic techniques were defined in 2007 by the Standing Committee for Sedation in Dentistry. ${ }^{5}$ The classification is shown in Table 1.

'Reader in Restorative Dentistry, School of Oral \& Denta Sciences, University of Bristol, Lower Maudlin Street, Bristol, BS1 2LY

Correspondence to: Dr Nigel Robb

Email:nigel.robb@bristol.ac.uk

\section{Refereed Paper}

Accepted 4 November 2013

DOI: $10.1038 /$ sj.bdj.2014.148

${ }^{\circledR}$ British Dental Journal 2014; 216: 223-227
In this paper the terms 'basic' and 'advanced' will be used.

The attitudes to the use of advanced techniques range widely within the medical and dental professions. At one end of the spectrum there are a group of practitioners who believe that using anything other than the basic techniques is tantamount to giving a general anaesthetic and should be either banned from clinical practice or limited to a hospital setting. At the other end of the spectrum are those who advocate the routine use of the advanced sedation techniques as the only sensible way forward as they are infinitely better than the standard techniques.

The truth lies somewhere between the two extremes. In adult patients the basic techniques are very effective and will allow the vast majority of patients to be managed both safely and effectively. Lecturers frequently report success rates in excess of 90\%. Although such claims are never backed up with published data, it is generally accepted that these estimates reflect clinical experience.

Since April 2001 in Scotland and 31 December 2001 in the rest of the United Kingdom, general anaesthesia for dentistry has been restricted to a hospital setting. ${ }^{10}$ Many services only provide paediatric exodontias, and those who do provide restorative dental care often only provide the most basic treatments that can be guaranteed to be successful. Thus, frequently endodontics and fixed prosthodontic treatments are not provided. Frequently aims are set as to an acceptable frequency of exposure to general anaesthesia. A figure of no repeat within five years is used in the special care dentistry service at Bristol Dental Hospital. This can result in potentially restorable teeth being extracted.

There is a need to be able to provide comprehensive care for those patients for whom standard techniques are not appropriate without having to resort to general anaesthesia with all the limitations on the care that can be provided.

It is implicit in the guidance (SDAC, ${ }^{1}$ SDCEP $^{4}$ and SCSD ${ }^{5}$ ) that the use of advanced sedation techniques must be justified. Such justification could be medical, dental, psychological or sedation-related. Examples are given in Table 2.

Within the Sedation Suite of Glasgow Dental Hospital and School and more recently in Bristol Dental Hospital there were a number of conscious sedation techniques provided by the author. These are listed in Table 3.

The following nine cases that we present are examples of where advanced sedation techniques have been used and have achieved success where basic techniques have been deemed to be inappropriate or have previously been unsuccessful.

Examples of two of the advanced techniques used by the author are given.

\section{PROPOFOL PATIENTS}

\section{Patient 1}

Midazolam sedation inappropriate due to 
potential pharmacokinetic interaction with concurrent medication.

A 14-year-old female patient was referred by her general dental practitioner (GDP) for treatment under sedation having failed sedation at a local sedation referral practice. The patient was taking long-term erythromycin for the control of acne and had a severe needle phobia relating to all injections. The patient had previously attended a sedation referral practice before commencing treatment with erythromycin, where oral sedation with midazolam had failed.

In view of the needle phobia oral sedation was felt to be the method of choice, with the possibility of proceeding to intravenous sedation if necessary. The concurrent use of erythromycin precluded the use of midazolam.

\section{Technique chosen}

Oral sedation with temazepam. As previous oral sedation had failed a dose of $40 \mathrm{mg}$ was chosen. If intravenous sedation were needed it was decided to use propofol Target Controlled Infusion (TCI) sedation.

\section{Treatment appointment}

Following recording of vital signs (blood pressure, heart rate and oxygen saturation) the patient was administered $40 \mathrm{mg}$ of temazepam elixir orally. The patient was monitored for 45 minutes. At that stage the patient was more relaxed than pre-sedation, but not sufficiently sedated for dental treatment. A 22 gauge cannula was placed in the dorsum of the right hand and TCI concentration of $1.5 \mu \mathrm{g} / \mathrm{ml}$ selected.

Once the target concentration had been achieved the patient happily accepted local anaesthesia with lidocaine 2\% with 1:80,000 adrenaline in both upper and lower right quadrants. Multiple plastic restorations were placed completing the patient's treatment in one appointment. The pulse and oxygen saturation were monitored throughout and remained within normal limits. The patient remained in verbal contact with the team throughout the treatment and recovery periods. Following treatment the patient was discharged to the care of her GDP for ongoing preventative advice and routine examinations.

\section{Patient 2}

Midazolam sedation inappropriate as patient taking high doses of benzodiazepines indicating tolerance to this group of drugs.

A 27-year-old male patient was referred by his GDP for treatment under sedation having failed sedation previously. He was dental phobic and particularly concerned about the presence of metal instruments in

Table 1 Basic and alternative conscious sedation techniques (standard and advanced conscious sedation techniques)

\begin{tabular}{|c|c|}
\hline Basic or standard & Alternative or advanced \\
\hline Inhalation sedation with nitrous oxide and oxygen & $\begin{array}{l}\text { Any form of conscious sedation for patients under } \\
\text { the age of } 12 \text { years }{ }^{\#} \text { other than nitrous oxide/oxygen } \\
\text { inhalation sedation }\end{array}$ \\
\hline $\begin{array}{l}\text { Intravenous sedation with a titrated dose } \\
\text { of midazolam }\end{array}$ & $\begin{array}{l}\text { Benzodiazepine + any other intravenous agent for } \\
\text { example: opioid, propofol and/or ketamine }\end{array}$ \\
\hline \multirow[t]{3}{*}{ Oral or transmucosal benzodiazepine sedation* } & $\begin{array}{l}\text { Propofol either alone or with any other agent for } \\
\text { example: benzodiazepine, opioid and/or ketamine }\end{array}$ \\
\hline & $\begin{array}{l}\text { Inhalational sedation using any agent other than } \\
\text { nitrous oxide/oxygen alone }\end{array}$ \\
\hline & $\begin{array}{l}\text { Combined (non-sequential) routes for example: } \\
\text { intravenous + inhalational agent }{ }^{+}\end{array}$ \\
\hline \multicolumn{2}{|c|}{$\begin{array}{l}\text { *Provided the sedationist competent in intravenous sedation } \\
\text { \#lt is recognised that the physical and mental development of individuals varies and may not necessarily correlate with } \\
\text { the chronological age } \\
+ \text { Except for the use of nitrous oxide/oxygen during cannulation }\end{array}$} \\
\hline
\end{tabular}

Table 2 Rationale for using alternative sedation techniques

\begin{tabular}{l|l|l|l} 
Medical & Psychological & Dental & Sedation \\
\hline $\begin{array}{l}\text { Concurrent medication } \\
\text { that interacts with sedative } \\
\text { for example, erythromycin } \\
\text { and midazolam }\end{array}$ & $\begin{array}{l}\text { Benzodiazepine } \\
\text { abuse that might } \\
\text { be reactivated by } \\
\text { therapeutic use }\end{array}$ & $\begin{array}{l}\text { Prolonged procedure } \\
\text { better managed with } \\
\text { continuous infusion } \\
\text { of drug }\end{array}$ & $\begin{array}{l}\text { Failed sedation with } \\
\text { basic techniques }\end{array}$ \\
\hline $\begin{array}{l}\text { Concurrent } \\
\text { benzodiazepine use } \\
\text { causing tolerance }\end{array}$ & $\begin{array}{l}\text { Short procedures to } \\
\text { avoid protracted recovery } \\
\text { periods }\end{array}$ & \\
\hline
\end{tabular}

\section{Table 3 Sedation techniques provided by the author}

\begin{tabular}{l|l} 
Basic (standard) techniques & Alternative (advanced) techniques \\
\hline Inhalation sedation with nitrous oxide/oxygen & Inhalation and intravenous sedation concurrently \\
\hline Intravenous midazolam sedation & Intravenous propofol TCl sedation \\
\hline Oral benzodiazepine sedation & Opioid and midazolam intravenous sedation \\
\hline Intranasal midazolam sedation & Oral midazolam and ketamine sedation \\
\hline $\begin{array}{l}\text { Inhalation for cannulation followed by intravenous } \\
\text { midazolam sedation }\end{array}$ & \\
\hline
\end{tabular}

his mouth. The patient had been using diazepam recreationally and therapeutically for a number of years and was taking $50 \mathrm{mg}$ per day at the time of referral. Previously sedation with midazolam in general dental practice had failed.

\section{Technique chosen}

It was decided to use intravenous sedation with TCI propofol.

\section{Treatment appointment}

Following recording of vital signs (blood pressure, heart rate and oxygen saturation) a 22 gauge cannula was placed in the dorsum of the left hand and TCI concentration of $1.5 \mu \mathrm{g} / \mathrm{ml}$ selected. Once the target concentration had been achieved the patient happily accepted local anaesthesia with lidocaine $2 \%$ with $1: 80,000$ adrenaline in all four quadrants of his mouth.
Following the administration of local anaesthesia, the patient became slightly oversedated and the TCI concentration was reduced to $1.3 \mu \mathrm{g} / \mathrm{ml}$. At that level, although the patient was quite talkative, cooperation was adequate to allow multiple plastic restorations to be placed. One extraction was deemed to be necessary, as well as some further periodontal treatment. A further appointment was arranged for this to be carried out. The pulse and oxygen saturation were monitored throughout. The patient had a tendency to breath hold and required frequent encouragement to breathe, which maintained a satisfactory oxygen saturation. The patient remained in verbal contact with the team throughout the treatment and recovery periods. Following treatment the patient was discharged to the care of his partner.

\section{Second treatment appointment}

When he attended the patient confirmed 
his satisfaction with the previous treatment. Following recording of vital signs (blood pressure, heart rate and oxygen saturation) a 22 gauge cannula was placed in the dorsum of the left hand and TCI concentration of $1.5 \mathrm{\mu g} / \mathrm{ml}$ selected. Once the target concentration had reached $1.3 \mu \mathrm{g} / \mathrm{ml}$ the patient happily accepted local anaesthesia with lidocaine $2 \%$ with $1: 80,000$ adrenaline for the extraction of a premolar tooth.

The extraction was completed successfully and a full mouth supra and subgingival scaling completed. The patient remained in verbal contact with the clinical team throughout the appointment, and following recovery was discharged into the care of his girlfriend.

\section{Patient 3}

Midazolam sedation inappropriate as patient taking high doses of benzodiazepines indicating tolerance to this group of drugs.

A 26-year-old male patient was referred by his GDP for treatment under sedation having failed sedation previously. He was dental phobic being anxious about all aspects of treatment. The patient had been using diazepam recreationally and therapeutically for a number of years and was taking $30 \mathrm{mg}$ per day at the time of referral. Previously sedation with midazolam had failed, as the patient had noticed no appreciable change in anxiety level after $20 \mathrm{mg}$ had been titrated.

\section{Technique chosen}

It was decided to use intravenous sedation with TCI propofol.

\section{Treatment appointments}

Following recording of vital signs (blood pressure, heart rate and oxygen saturation) a 22 gauge cannula was placed in the dorsum of the left hand and TCI concentration of $1.5 \mathrm{\mu g} / \mathrm{ml}$ selected. Once the target concentration had been achieved the patient happily accepted local anaesthesia with lidocaine $2 \%$ with $1: 80,000$ adrenaline in both upper quadrants of his mouth.

Following the administration of local anaesthesia, the patient's cooperation was adequate to allow multiple plastic restorations to be placed. A further appointment was arranged for completion of his treatment. The pulse and oxygen saturation were monitored throughout and remained within normal limits. The patient remained in verbal contact with the team throughout the treatment and recovery periods. Following treatment the patient was discharged to the care of his partner.

The second treatment appointment followed the same clinical course as the first, with the exception that the patient required a TCI concentration of $1.7 \mu \mathrm{g} / \mathrm{ml}$ to allow treatment to be completed.

\section{Patient 4}

Midazolam sedation inappropriate as patient reported abnormally protracted recovery from this type of sedation.

A 64-year-old female patient was referred from one of the consultants within the periodontal department of the dental hospital to have an area of external resorption affecting a lower incisor tooth repaired. The patient was assessed as suitable for intravenous sedation. There was no relevant medical history and the baseline blood pressure and pulse were within acceptable limits. Written consent for treatment was obtained.

\section{Treatment appointment}

The patient was sedated with a titrated dose of $7 \mathrm{mg}$ of midazolam, following which local anaesthesia was administered and treatment carried out uneventfully. The patient's pulse remained within $10 \%$ of the presedation value and oxygen saturation remained within 3\% of the presedation value throughout the period of observation.

The patient had a normal recovery under the supervision of a sedation trained dental nurse and was discharged into the care of her husband approximately 20 minutes following the completion of the dental treatment.

Subsequently the patient was referred for nonsurgical periodontal treatment under intravenous sedation. At assessment the patient reported that it had taken approximately three days for her to recover from the intravenous sedation that she had previously received. After some discussion of the options the patient consented to receive intravenous propofol sedation.

\section{Treatment appointment}

Following recording of vital signs (blood pressure, heart rate and oxygen saturation) a 22 gauge cannula was placed in the dorsum of the left hand and TCI concentration of $1.5 \mu \mathrm{g} / \mathrm{ml}$ selected. As the blood concentration approached $1.0 \mu \mathrm{g} / \mathrm{ml}$, the patient showed signs of adequate sedation and agreed to the start of dental treatment. The target concentration was altered to maintain a concentration of $1.0 \mu \mathrm{g} / \mathrm{ml}$. The administration of local anaesthesia and dental treatment was carried out uneventfully. The patient's pulse remained within 10\% of the presedation value and oxygen saturation remained within 3\% of the presedation value throughout the period of observation. The patient had a normal recovery under the supervision of a sedation trained dental nurse and was discharged into the care of her husband approximately ten minutes following the completion of the dental treatment. At review the patient indicated that she had recovered from the propofol sedation much more rapidly than from midazolam sedation, and that the day after treatment had felt completely recovered. The patient remained in verbal contact with the team throughout the treatment and recovery periods at both sedation appointments.

\section{PATIENTS TREATED WITH A COMBINATION OF OPIOID AND MIDAZOLAM}

\section{Patient A}

Midazolam alone achieved sedation but without sufficient anxiolysis to allow dental treatment to be completed successfully.

A 17-year-old male patient was referred for treatment under intravenous sedation following an unsuccessful episode of intravenous sedation in general dental practice. The patient was unable to recall any details of the treatment appointment, but the referral letter indicated that despite the patient appearing to be sedated to a satisfactory level, cooperation with treatment was so poor that the dental treatment had to be aborted.

Medically the patient was fit and well with no relevant medical history. The patient was anxious about all aspects of dental treatment, but reported that intraoral injections and tooth preparation were the most frightening for him. The patient required multiple intracoronal plastic restorations in all four quadrants.

After discussion with the patient and his mother it appeared that despite having an adequate level of sedation, the patient was insufficiently anxiolysed to undergo dental treatment. Thus it was decided to use a combination of fentanyl and midazolam.

\section{Treatment appointments}

The patient required five sedation appointments to complete the dental treatment. On each occasion, following recording of vital signs (blood pressure, heart rate and oxygen saturation) a 22 gauge cannula was placed in the dorsum of the right hand and a dose of $50 \mu \mathrm{g}$ was administered. Midazolam was then titrated to clinical effect. The doses required for the five appointments were: $20 \mathrm{mg}, 18 \mathrm{mg}, 19 \mathrm{mg}, 20 \mathrm{mg}$ and $19 \mathrm{mg}$.

The patient remained in verbal contact with the dental team at all times throughout all appointments. At no stage during any appointment did the patient's oxygen saturation drop below 98\%. The patient did require a significant amount of reassurance and behavioural management, but cooperation with dental treatment was good.

Following completion of his treatment, the patient was discharged to the care of his mother. At each subsequent appointment 
both patient and mother expressed their satisfaction with previous treatment.

\section{Patient B}

Midazolam alone achieved sedation but without sufficient anxiolysis to allow dental treatment to be completed successfully.

A 24-year-old female patient of the Dental Hospital had undergone treatment under intravenous sedation with midazolam alone with titrated doses ranging between 9 and $15 \mathrm{mg}$. At these appointments the patient had been tearful and cooperation had been poor. The patient's relevant medical history was hypothyroidism, panic attacks and an allergy to erythromycin. Current medication was thryoxine, cipirelex and an oral contraceptive. The patient was judged to be ASA II.

Following the poor compliance with treatment under midazolam sedation a decision was made to use a combination of fentanyl and midazolam.

\section{Treatment appointments}

Following recording of vital signs (blood pressure, heart rate and oxygen saturation) a 22 gauge cannula was placed in the dorsum of the right hand and a dose of $50 \mu \mathrm{g}$ was administered. Midazolam was then titrated to clinical effect. A dose of $10 \mathrm{mg}$ was used. Dental treatment was completed without any crying from the patient. Cooperation was excellent and the oxygen saturation and pulse remained within normal limits throughout the procedure. The patient remained in verbal contact with the team throughout the treatment and recovery periods.

\section{Patient C}

High doses of midazolam used alone were required and only achieved limited cooperation.

A 31-year-old female was referred for dental treatment having had failed intravenous sedation in both general dental practice and the Community Dental Service. There was no relevant medical history, but the patient reported that she had suffered emotional problems subsequent to a physical assault.

\section{Dental treatment required}

The patient required a scale and polish, five plastic restorations, re-root treatment of an upper central incisor and replacement of porcelain laminate veneers on the upper anterior teeth.

\section{Treatment appointments}

An initial attempt was made to treat the patient with midazolam alone, but despite titrated doses of $24 \mathrm{mg}$ and $20 \mathrm{mg}$ of midazolam the patient remained very anxious about treatment and cooperation was limited. A decision was reached to use a combination of opioid and midazolam. As the patient's treatment commenced in 2003 the drug nalbuphine was selected as the opioid of choice.

The patient underwent sedation with an initial dose of $10 \mathrm{mg}$ nalbuphine followed by a titrated dose of midazolam. The dose range of midazolam used was between 9 and $10 \mathrm{mg}$. On one occasion there was a transient dip in oxygen saturation to $94 \%$, which was managed by encouraging the patient to breathe. At the remaining appointments the oxygen saturation remained at $98 \%$ or above throughout the treatment. At all the appointments the patient's cooperation was good and the patient was visibly more relaxed than with midazolam sedation.

In 2007, after nalbuphine had been withdrawn from sale in the UK, the patient was sedated with a dose of $50 \mu \mathrm{g}$ fentanyl followed by a titrated dose of midazolam. The patient was sedated using this combination of agents ten times. The titrated dose of midazolam ranged from 13-24 mg - the latter dose only being used on one occasion when the patient was particularly stressed by her domestic circumstances. On all the other occasions the dose was less than $20 \mathrm{mg}$. At all appointments the oxygen saturation remained within normal limits, and the lowest that was recorded was 97\%. The dental treatment was completed with good cooperation. The patient remained in verbal contact with the team throughout the treatment and recovery periods during all of the treatment appointments, irrespective of the sedation technique used.

\section{Patient D}

Midazolam alone achieved sedation but without sufficient anxiolysis to allow dental treatment to be completed successfully.

In 2003 a 17-year-old male patient had been referred for treatment under sedation. The patient was ASA I with no relevant medical history.

\section{Treatment appointment}

The patient received treatment under intravenous midazolam sedation where titrated doses of $10 \mathrm{mg}$ were given initially. At one of the appointments incremental top ups totalling $4 \mathrm{mg}$ were given. The treatment was completed at both appointments, but the operating conditions and cooperation were poor.

In 2007 the patient was re-referred for treatment. There was no change in the medical history.

\section{Treatment appointments}

Initially treatment was attempted under midazolam sedation with titrated doses of 11-19 mg given at different appointments. At one of the appointments the patient started to hyperventilate mid treatment. At all appointments the oxygen saturation remained at $100 \%$.

A decision was made to manage the patient using fentanyl and midazolam. The patient had restorative dental treatment carried out over two appointments. At both appointments he was given $50 \mu \mathrm{g}$ fentanyl followed by a titrated dose of midazolam. At the first appointment $9 \mathrm{mg}$ of midazolam was used as an initial dose followed by an additional $5 \mathrm{mg}$ in increments. At the second appointment a titrated dose of $15 \mathrm{mg}$ midazolam was used. In both cases this allowed the dental treatment to be completed uneventfully. The patient's oxygen saturation remained above 97\% throughout and there was no recurrence of the episode of hyperventilation. The patient remained in verbal contact with the team throughout the treatment and recovery periods.

\section{Patient E}

Midazolam alone achieved sedation but without sufficient anxiolysis to allow dental treatment to be completed successfully.

In 2003 an 18-year-old female was referred for extractions under sedation. The patient suffered from mild asthma and used a salbutamol occasionally. The patient was assessed to be ASA II.

\section{Treatment appointments}

The treatment was carried out over two appointments with titrated doses of $9 \mathrm{mg}$ and $10 \mathrm{mg}$ midazolam. At both appointments the patient was obviously distressed and cooperation was poor.

The patient was subsequently re-referred in 2008, at which point there had been no change in her medical history.

Treatment was initially attempted with intravenous midazolam sedation, but despite a titrated dose of $17 \mathrm{mg}$ the treatment had to be aborted due to poor cooperation. A decision was made to treat the patient with a combination of fentanyl and midazolam. The patient's treatment was completed over two appointments. On each occasion an initial dose of $50 \mu \mathrm{g}$ of fentanyl was given followed by a titrated dose of midazolam (12 $\mathrm{mg}$ and $9 \mathrm{mg}$ ). The patient's oxygen saturation and pulse remained within normal limits throughout. Patient cooperation was excellent.

At a third referral in 2011, again the patient's medical history was unchanged. Treatment was completed at a single appointment with an initial dose of $50 \mu \mathrm{g}$ of fentanyl followed by a titrated dose of 
$10 \mathrm{mg}$ of midazolam. The patient remained in verbal contact with the team throughout the treatment and recovery periods.

\section{DISCUSSION}

The cases reported here represent a minority of the patients referred for conscious sedation. The vast majority of adult patients who are referred can be managed with either a combination of nitrous oxide and oxygen or the use of midazolam by whichever is the most appropriate route for that patient. In these cases midazolam sedation had been ineffective, and given the patients' levels of anxiety, inhalation sedation was deemed to be inappropriate.

The common feature in cases 2 and 3 managed with propofol was that the patients had a tolerance to benzodiazepines secondary to chronic addiction, making the use of midazolam inappropriate. In the other two cases there was a problem with prolonged recovery. In one case this was idiopathic, but in the other it could have been due to a pharmacokinetic interaction between the sedative and the patient's prescribed medication.

The patients who were managed with a combination of opioid and midazolam had all shown signs of an adequate level of sedation when they had received titrated doses intravenously. In all the cases, however, when treatment was attempted it was apparent that there was an insufficient level of anxiolysis to allow completion of treatment.
One possible explanation for this is that the sedative and anxiolytic effects of the benzodiazepines are produced via different receptor sites in the central nervous system. Sedation is produced via the GABA (gamma amino butyric acid) receptor complex in the cerebral cortex and anxiolysis via the glycine receptor complex in the brainstem. Thus increases in these clinical effects do not necessarily parallel each other as drug dose increases. Clinicians who are experienced in the use of conscious sedation will be familiar with the patient who, when 'sedated' with midazolam shows little sign of sedation, but is entirely happy for treatment to be undertaken. They will also be familiar with the patient who looks sedated, almost to the point of losing verbal contact, but who claims to still be frightened of the treatment.

\section{CONCLUSION}

The judicious use of these techniques has allowed patients who were unmanageable with the basic sedation techniques to receive dental treatment under sedation rather than having to resort to general anaesthesia. All of the patients reported here were entirely satisfied with their treatment under the advanced techniques, but not at all comfortable with treatment under intravenous midazolam.

These techniques should be made available to those patients who need this form of management.
1. Standing Dental Advisory Committee (SDAC). Conscious sedation in the provision of dental care. Report of an Expert Group on Sedation for Dentistry. 2003.

2. The Royal College of Anaesthetists. Standards and Guidelines for General Anaesthesia for Dentistry. February 1999.

3. Implementing and Ensuring Safe Sedation Practice for Healthcare Procedures in Adults. London: Academy of Medical Colleges and their Faculties, 2001.

4. Scottish Dental Clinical Effectiveness Programme. Conscious sedation in dentistry, 2nd ed. June 2012. Online article available at www.sdcep.org.uk/index. aspx?o=2331 (accessed 14 November 2013).

5. Faculty of Dental Surgery. The Royal College of Surgeons of England. Standards for Conscious Sedation in Dentistry: Alternative Techniques. A Report from the Standing Committee on Sedation for Dentistry. October 2007.

6. The Dental Sedation Teachers Group. Training in Conscious Sedation for Dentistry. 2005. Online article available at www.dstg.co.uk/documents/ (accessed February 2014).

7. Independent Expert Group on Training and Standards for Sedation in Dentistry. Advanced Conscious Sedation Techniques for Adult Dental Patients: Training Syllabus. September 2011. Online information available at www.saad.org.uk/ wp-content/uploads/2012/11/Adult-Sylabus-TextCropped.pdf (accessed February 2014).

8. Independent Expert Group on Training and Standards for Sedation in Dentistry. Advanced Conscious Sedation Techniques for Paediatric Dental Patients: Training Syllabus. December 2011 Online information available at www.saad.org.uk/ wp-content/uploads/2012/11/Paediatric-syllabusfor-website.pdf (accessed February 2014).

9. Independent Expert Group on Training and Standards for Sedation in Dentistry. A Guide to Maintaining Professional Standards in Conscious Sedation for Dentistry. September 2011. Online information available at www.saad.org.uk/ wp-content/uploads/2012/11/CPD-Document-TextCropped.pdf (accessed February 2014).

10. Department of Health. A conscious decision: a review of the use of general anaesthesia and conscious sedation in primary dental care. 1 July 2000.

\section{Corrigendum}

\section{General article (BDJ 2014; 216: 83-87)}

The 'Dental Institution' in London, 1817-21. A prototype dental school: the vision of Levi Spear Parmly' In the above General article, Lilian Lindsay was incorrectly referred to as a Dame. Her correct appointment was CBE.

The author apologises for any confusion caused. 